\title{
Athéisme, scepticisme et doute religieux au Moyen Âge.
}

Notes de lecture à propos de trois publications récentes

\section{Thomas Lienhard}

\section{(2) OpenEdition}

\section{Journals}

Édition électronique

URL : http://journals.openedition.org/ifha/194

DOI : $10.4000 /$ ifha. 194

ISSN : 2198-8943

Éditeur

IFRA - Institut franco-allemand (sciences historiques et sociales)

Édition imprimée

Date de publication : 6 février 2011

Pagination : 188-205

ISSN : 2190-0078

Référence électronique

Thomas Lienhard, «Athéisme, scepticisme et doute religieux au Moyen Âge. », Revue de l'IFHA [En ligne], 3 | 2011, mis en ligne le 16 février 2012, consulté le 19 avril 2019. URL : http:// journals.openedition.org/ifha/194; DOI : 10.4000/ifha.194

Ce document a été généré automatiquement le 19 avril 2019

(CIFHA 


\title{
Athéisme, scepticisme et doute religieux au Moyen Âge.
}

Notes de lecture à propos de trois publications récentes

\author{
Thomas Lienhard
}

\section{NOTE DE L'ÉDITEUR}

Thomas Lienhard a été directeur de la MHFA puis de l'IFHA de septembre 2007 à août 2011.

Dans un numéro récent de la présente Revue, on signalait l'absence d'études satisfaisantes concernant le scepticisme religieux au Moyen Âge, et on annonçait la préparation d'une rencontre scientifique destinée à pallier cette lacune ${ }^{1}$. Dans l'intervalle, toutefois, trois nouvelles publications ont fait progresser nos connaissances en ce domaine, de sorte que, à défaut d'un colloque, on voudrait présenter ici les principaux résultats ainsi obtenus ${ }^{2}$. Rappelons l'enjeu essentiel du débat: les trois ouvrages en question partagent, jusque dans leur titre, le souhait de réviser l'a priori selon lequel l'époque médiévale aurait été l'âge de la foi par excellence et, de ce fait, incompatible avec le scepticisme ou le doute religieux, a fortiori avec l'athéisme. Ce schéma du Moyen Âge engoncé dans une foi chrétienne universelle ne constitue pas seulement, en effet, une image d'Épinal préscientifique. Lucien Febvre également, dans son célèbre Problème de l'incroyance au XVI ${ }^{e}$ siècle, estimait ainsi que l'incroyance n'était pas accessible aux mentalités médiévales ${ }^{3}$; quant à l'athéisme proprement dit, la majorité des auteurs considère que son apparition dans l'histoire européenne doit être datée du XVIIe siècle avec d'Holbach, en aucun cas avant l'apparition du terme d'atheus au XVIe siècle. Ces opinions furent certes remises en question récemment par Georges Minois ${ }^{4}$, mais pour ce qui concerne la période médiévale, la démonstration restait à faire.

2 C'est désormais chose faite, ou du moins des progrès considérables ont-ils été permis par les trois ouvrages analysés ici. Avant d'en présenter les résultats, commençons par 
indiquer quel est l'objet exact de ces trois publications, car si celles-ci étudient certes un thème similaire, elles divergent par l'ampleur de leur approche. Ainsi, Peter Dinzelbacher s'intéresse exclusivement à l'athéisme proprement dit ; pour sa part, Dorothea Weltecke élargit le champ d'étude à tous les doutes dogmatiques ou ecclésiologiques; enfin, Sabina Flanagan prend en compte l'ensemble des doutes médiévaux possibles, depuis le domaine du christianisme jusqu'aux incertitudes judiciaires, aux inquiétudes à propos de l'avenir, aux angoisses d'ordre financier, etc. - dans le présent article, on ne retiendra toutefois de son étude, par souci de cohérence, que les aspects qui ont trait au domaine religieux. Ces trois études ont ainsi en commun de ne pas prendre en compte le phénomène de l'hérésie, se concentrant au contraire sur les personnages médiévaux qui, tout en restant dans le giron catholique, ont exprimé leurs doutes à propos de cette foi ou de cette institution, ou encore de ceux qui ont remis en cause le christianisme au point de ne plus reconnaître aucune divinité. Elles partagent également un intervalle chronologique semblable, limité à un long XIIe siècle dans le cas de S. Flanagan, élargi à tout le bas Moyen Âge dans le cas des deux publications germanophones, sans qu'aucun auteur ne prenne en compte le haut Moyen Âge ni l'époque moderne. À côté de ces points communs, signalons enfin une différence de genre entre les trois études : alors que P. Dinzelbacher et $\mathrm{S}$. Flanagan proposent une brève synthèse orientée vers la recherche de modèles théoriques (par ailleurs très engagés, chantant l'histoire de l'athéisme comme un vecteur progressiste), D. Weltecke offre un ouvrage plus volumineux qui, tout en étant très précis dans ses concepts, se tourne davantage vers l'étude de sources originales. Telles sont donc les caractéristiques de cette salve éditoriale : que nous apporte celle-ci à propos de l'athéisme, du scepticisme et du doute religieux au Moyen Âge?

Un des principaux mérites de ces ouvrages consiste à passer au peigne fin l'historiographie consacrée au scepticisme médiéval. D'une part, P. Dinzelbacher s'efforce d'évaluer les enjeux actuels de cette historiographie, considérant que la rareté présente des études consacrées à ce thème est à mettre en relation avec le recul de la laïcité dans l'Union européenne depuis quelque vingt ans : en construisant un Moyen Âge totalement voué au christianisme, on produirait ainsi un modèle (pseudo-)historique pour justifier l'extension de la sphère religieuse aujourd'hui ${ }^{5}$. D'autre part, D. Weltecke souligne, au contraire, les a priori des auteurs qui, depuis le début de l'époque moderne, se sont penchés sur l'historiographie du scepticisme et particulièrement de l'athéisme ${ }^{6}$. Née dans le contexte des conflits confessionnels du XVIe siècle, cette historiographie a d'abord inséré l'athéisme dans la liste des déviances médiévales que l'on souhaitait condamner : protestants et catholiques s'accusaient ainsi mutuellement d'avoir favorisé ce prétendu vice, avant que les premiers, lors d'un renversement intellectuel survenu au XVIIe siècle, ne s'attribuent progressivement les sceptiques médiévaux comme des ancêtres spirituels, opposés au catholicisme et persécutés comme ils estimaient l'être eux-mêmes. Par la suite, le thème de l'athéisme médiéval fut encore ballotté entre le Kulturkampf et les conflits sociaux du XIXe siècle, les historiens croisant alors le fer pour déterminer si ce courant intellectuel avait été porté d'abord par les élite sociales ou par le petit peuple; le courant marxiste, en particulier, joua un rôle important en ce domaine jusqu'à l'époque de la RDA, associant le développement de l'athéisme avec l'inéluctable processus de libération du prolétariat, établissant une ligne diachronique depuis l'hérésie vers le panthéisme, puis vers l'athéisme matérialiste, et proposant enfin une galerie héroïque de pionniers médiévaux de l'athéisme, à laquelle les historiens de tout bord se référèrent longtemps pour aborder ce sujet. Ainsi, parce qu'elle véhiculait un héritage 
particulièrement ancien, cette historiographie était restée exceptionnellement polémique jusque dans les dernières années du XXe siècle.

4 Parmi les trois ouvrages recensés, celui de D. Weltecke fait preuve d'une acribie particulière vis-à-vis de cette historiographie, en revenant méthodiquement sur la galerie des héros de l'athéisme citée de manière récurrente par les historiens de ce domaine. L'auteur reprend ainsi en profondeur le dossier du comte Jean de Soissons décrit par Guibert de Nogent (1053-1124), auquel les sources prêtent des propos ironiques sur la Résurrection et sur l'utilité de la messe ${ }^{7}$; de l'empereur Frédéric II, souvent évoqué pour son conflit avec le pape Innocent IV et plus précisément pour une phrase qui lui fut attribuée, selon laquelle Moïse, Jésus et Mahomet n'étaient que d'habiles imposteurs ${ }^{8}$; de la révolte des paysans anglais en 1381, qui prit parfois un tour anticlérical et à propos de laquelle plusieurs sources évoquèrent l'incroyance des élites sociales dans les régions concernées ${ }^{9}$; de l'impératrice Barbara de Cilli (1392-1451), parfois dénoncée comme athée et (partant ?) comme lubrique par les sources de son époque ${ }^{10}$; des intellectuels qui, dans la France du second XIIIe siècle, débattirent du dogme chrétien avec des positions que l'on qualifia d'averroïstes ${ }^{11}$; des paysans de Montaillou, enfin, qui, à la fin du XIIIe siècle, se montrèrent réticents en particulier envers les notions d'Incarnation et de résurrection des corps ${ }^{12}$.

Or, à l'opposé de l'historiographie traditionnelle, D. Weltecke relativise énergiquement la portée de ces exemples, considérant qu'on n'y trouve aucun facteur d'assurance à propos d'un scepticisme radical, a fortiori à propos d'une forme d'athéisme au Moyen Âge. Elle rappelle, en effet, que les sources employées pour ces dossiers sont presque systématiquement très orientées, et élaborées contre le personnage concerné pour des raisons qui n'avaient rien à voir avec l'orthodoxie religieuse. Même lorsqu'il ne s'agissait pas de nuire à la réputation de celui que l'on présentait comme incroyant, de nombreux autres projets d'écriture pouvaient entrer en compte, qui incitent tout autant à la méfiance : ainsi, constatant que la présentation de Jean de Soissons ressemble fort à une polémique antijuive, D. Weltecke estime qu'un tel comportement judaïsant était en soi fort peu probable au XIe siècle, mais que la source en question souhaitait peut-être dévaloriser indirectement le judaïsme en associant celui-ci avec un personnage impopulaire, celui du comte. Plus généralement, les accusations d'incroyance étaient souvent trop incohérentes pour être crédibles, attribuant notamment aux intéressés des croyances résolument contradictoires, qui ressemblaient à une compilation maladroite des topoi traditionnellement employés contre l'hérésie. S'ajoutent à cela des malentendus linguistiques par lesquels les historiens ont parfois interprété comme une preuve d'athéisme des expressions qui, comme celle d'impietas, dénonçaient simplement un caractère asocial. Au terme de ce travail de laminage méticuleux, les réticences religieuses médiévales que l'on peut effectivement retenir dans cette série d'exemples ne portent que sur des points très partiels du dogme catholique, tels que la réalité des miracles ou l'efficacité de tel ou tel rituel religieux. La grande lignée héroïque souvent invoquée pour l'histoire du scepticisme religieux offre ainsi, à en croire D. Weltecke, une excellente source pour étudier divers conflits sociaux du second Moyen Âge; mais d'athéisme, point.

6 Faut-il donc en revenir à la thèse de Lucien Febvre, et considérer que les esprits médiévaux n'étaient pas en mesure de concevoir le scepticisme religieux? Bien au contraire, les trois ouvrages présentés ici surprennent non seulement par la prolifération des réactions médiévales méfiantes envers des points parfois majeurs du christianisme, 
mais également par la décontraction avec laquelle ces réticences étaient présentées par ceux qui les exprimaient et par les auteurs qui les ont rapportées.

Il est vrai que dans certains cas, le récit semble avoir eu une fonction purement théorique, ne reflétant pas les opinions réelles du Moyen Âge. C'est sans doute ainsi qu'il faut émousser le tranchant apparent d'un récit dans lequel Pierre le Vénérable (vers 1092-1156) affirma que, lors d'une vision nocturne, sa plus grande inquiétude avait consisté à savoir si la foi chrétienne à laquelle il avait consacré sa vie jusque-là était bien la bonne ${ }^{13}$; un cas similaire est celui d'Otloh de Saint-Emmeram auquel des démons posèrent la même question ${ }^{14}$. Dans la même catégorie, on peut évoquer les débats intellectuels cherchant à élaborer les preuves de l'existence de Dieu, dans lesquels un contradicteur défend la thèse opposée ${ }^{15}$. Il faut toutefois signaler que dans les trois exemples que l'on vient d'évoquer, c'est la thèse orthodoxe qui l'a emporté dans l'esprit de tous les assistants. Surtout, S. Flanagan, P. Dinzelbacher et D. Weltecke rappellent de concert la fonction pédagogique de tels écrits. Ainsi, dans le cas des témoignages produits par Pierre le Vénérable et par Otloh, donc par un abbé et un écolâtre, il s'agissait de prévenir les jeunes moines contre le scepticisme et de leur enseigner les outils pour s'en prémunir; notons d'ailleurs que les auteurs prenaient soin de ne s'attribuer de tels doutes dangereux que dans le cadre littéraire bien réglé de la vision, et non pas de leurs opinions raisonnées. Quant aux débats théoriques sur l'(in)existence de Dieu, ils répondaient à un souci logique de la scolastique, soucieuse de confronter toute proposition avec son contraire, et vraisemblablement pas à la nécessité de convaincre des sceptiques bien réels. Cette première catégorie de textes montre ainsi que l'hypothèse d'une possible fausseté du dogme chrétien pouvait certes être envisagée, écrite et discutée dans la seconde moitié du Moyen Âge, ce qui constitue une pierre de touche importante; mais elle ne plaide en rien pour l'existence effective d'un scepticisme actif chez des esprits médiévaux.

D'autres dossiers, en revanche, échappent à cette grille de lecture critique et semblent donc refléter des (in)croyances réellement diffusées dans la société médiévale. On a déjà évoqué plus haut le cas des villageois de Montaillou interrogés par l'Inquisition : dans ce type de situations, il est difficile de croire à une fiction théorique, car les actes de ces interrogatoires n'avaient pas vocation à être diffusés auprès d'un grand public, de sorte que les diverses hésitations religieuses qui y sont consignées peuvent être reçues sans réserve. Il en va de même pour un autre dossier particulièrement remarquable ${ }^{16}:$ dans ses Dialogues au sujet des miracles, Césaire de Heisterbach (v. 1180-v. 1240) rapporte ainsi le cas d'une nonne qui, peu de temps après son entrée au monastère, regretta sa décision et déclara ne plus croire en l'existence de Dieu, des anges ni du Ciel. Césaire s'efforce certes d'expliquer ce basculement par des interventions démoniaques, mais ne cherche pas à passer le phénomène sous silence. Surtout, il n'ajoute pas une fin édifiante à cet épisode : dans son récit, la nonne réchappe certes d'une tentative de suicide, mais rien n'est dit à propos d'un retour à l'orthodoxie religieuse, ce qui semble exclure toute fonction pédagogique ou moralisatrice dans cet extrait ${ }^{17}$. Celui-ci constitue ainsi l'un des témoignages les plus explicites et les plus incontestables pour l'existence d'un athéisme médiéval.

9 Il est vrai qu'à côté de ce cas extrême, le scepticisme religieux pouvait adopter des formes et des degrés très divers; et après l'efficace relecture des sources proposée par les trois études que l'on présente ici, il n'est peut-être pas inutile de proposer une typologie des remises en question médiévales dans le domaine religieux. La forme la plus fréquemment 
attestée dans les sources est incontestablement l'anticléricalisme, qui rejette soit la puissance institutionnelle de l'Église (en particulier les prélèvements matériels effectués par celle-ci), soit l'efficacité de certains rituels pratiqués par le clergé. On peut y associer les réactions consistant à reconnaître l'autorité des Évangiles, mais à rejeter celle de la Tradition catholique. À propos de ces deux premiers cas de figure, P. Dinzelbacher rappelle à juste titre le caractère anachronique d'un réflexe qui consisterait à y voir des prototypes de l'athéisme : en règle générale, de telles réactions religieuses émanaient au contraire de personnes particulièrement pieuses, soucieuses d'aboutir à un christianisme épuré parce que réformé ${ }^{18}$. Il est vrai que cette différence de degré entre l'anticléricalisme et l'athéisme n'était pas nécessairement perçue comme telle par l'Église médiévale, qui, en pratique, pourchassait l'un avec autant d'âpreté que l'autre ; mais pour l'historien moderne soucieux d'échapper à une vision téléologique de l'athéisme, il peut être utile de distinguer soigneusement - ce ne fut pas toujours le cas - entre les exigences médiévales de réforme et un sentiment consistant à rejeter l'existence même de Dieu.

D'autres cas en revanche, même s'ils ne sont pas associés avec une profession d'athéisme explicite, témoignent pourtant d'un degré bien plus fort de réticence à l'égard de l'existence ou de la puissance du divin. Tel est en particulier le cas du scepticisme envers la théodicée, fréquemment attesté dans les sources, consistant à réfuter la toutepuissance de Dieu au nom de l'existence du mal ${ }^{19}$. On peut y associer une autre forme de remise en cause, étonnamment moderne, consistant à miner le dogme chrétien en considérant que celui-ci, à défaut d'être vrai, avait principalement une fonction de régulation sociale : quatre siècles avant Pomponazzi, cette conception cynique était déjà portée par un prêtre anonyme, décrit par Giraud de Barri au XIIe siècle ${ }^{20}$. Partielles ou totales, toutes les formes de remise en cause du dogme catholique ont ainsi trouvé leurs porte-voix dans la seconde moitié du Moyen Âge.

11 La présence du scepticisme étant ainsi attestée, il reste à en identifier les facteurs. Or, pour ce faire, une longue vague historiographique a tenté d'expliquer le doute, comme on l'avait déjà fait pour l'hérésie, en invoquant les influences venues d'autres cultures, en particulier les échanges avec le judaïsme et avec l'islam. Là encore, les trois ouvrages que l'on présente ici fournissent une utile mise au point : pas plus que pour le développement de l'hérésie, les contacts avec d'autres religions ne semblent avoir joué un rôle déterminant pour l'évolution du scepticisme. Certes, les doutes religieux exprimés par Herbert de Bosham (†1186) furent probablement influencés par la lecture, directe ou indirecte, de Rashi ${ }^{21}$. Mais ce cas semble être resté exceptionnel, et c'est plutôt une causalité inverse qui ressort des dernières publications à ce sujet: des dissensions croissantes parmi les chrétiens auraient conduit l'Église, moins assurée de son autorité qu'auparavant, à durcir ses positions envers les juifs, soupçonnés de vouloir profiter de cet état de faiblesse, ce qui expliquerait en partie la simultanéité de l'hérésie, du scepticisme et de l'antijudaïsme dans la seconde moitié du Moyen Âge ${ }^{22}$.

Ce n'est donc pas en recherchant des influences extérieures que l'on trouvera les causes du scepticisme durant cette période. De ce fait, les études les plus récentes se sont tournées vers des données sociologiques internes au christianisme, en particulier vers l'évolution des groupes sociaux et des structures d'enseignement. Le schéma dominant était le suivant: sous l'influence nouvelle de la scolastique, naturellement portée à la remise en question des systèmes intellectuels antérieurs, les élites sociales de l'Occident médiéval auraient peu à peu exprimé des idées sceptiques, voire franchement athées ; et ce serait sous l'influence modernisatrice de ces élites que l'ensemble de la population, au 
seuil de l'époque moderne, aurait été gagnée par ces idées. Ce schéma sociologique était déjà en partie admis par des auteurs médiévaux comme Jean de Salisbury, qui estimait que les lettrés étaient plus facilement sujets au doute ${ }^{23}$; on connaît notamment l'accusation explicite de Bernard de Clairvaux envers Abélard, auquel il reprochait de favoriser l'incroyance par son approche scolastique ${ }^{24}$. Et de fait, ce modèle explicatif semble corroboré par une certaine tolérance vis-à-vis de l'athéisme dans les strates supérieures de la société, comme le prouve l'exemple, parmi d'autres, du médecin de Louis IX, à propos duquel Guillaume de Chartes écrit avec bienveillance que le malheureux souffrait d'une bile qui l'empêchait de croire en la divinité de Jésus ${ }^{25}$. Ce schéma d'évolution par les élites semblait donc cohérent, et parmi les trois auteurs que l'on présente ici, P. Dinzelbacher y reste fermement attaché ${ }^{26}$.

En revanche, S. Flanagan et D. Weltecke s'en détachent, pour deux raisons principales. En premier lieu, l'une et l'autre rappellent le fait que la scolastique n'avait pas pour vocation de tout mettre en doute, mais au contraire de préciser les connaissances disponibles, et cela uniquement pour des domaines bien délimités. Les règles et les bornes de cet exercice sont explicitement présentées par Abélard (soucieux de se blanchir, il est vrai, du soupçon d'incitation à la déviance) dans le Sic et non ${ }^{27}$ : selon lui, c'est l'existence primordiale d'un doute qui suscite les interrogations, et celles-ci qui conduisent à la vérité. Dans cette perspective, pour les sujets qui font déjà l'objet d'un consensus parmi les sages, il est vain de se poser des questions supplémentaires, et c'est seulement dans le cas d'une lacune ou d'une contradiction dans le corpus des autorités que le questionnement scolastique peut faire du sens. On est ainsi bien loin d'un relativisme universel: selon cette grille d'analyse, il devenait certes loisible de sonder la toutepuissance ou l'omniscience de Dieu, mais aucunement de poser la question de l'existence divine, alors que ce dernier débat avait déjà été osé, bien avant le développement de la scolastique, dans le cadre de discussions monastiques du haut Moyen Âge. Si l'on retient cette position d'Abélard, la scolastique a précisé les connaissances et renforcé les convictions, dans le cadre de schémas intellectuels déjà établis, bien plus qu'elle n'a suscité le doute ni, a fortiori, l'athéisme.En second lieu, une autre raison qui incite S. Flanagan et $\mathrm{D}$. Weltecke à la prudence envers l'idée d'une influence des élites réside dans le fait que, après une efficace critique des sources comme celle qui a été décrite plus haut, la majorité des signes recevables de scepticisme médiéval provient de catégories sociales intermédiaires, voire modestes. Sans surprise, on n'y lira pas de longs argumentaires théoriques; en revanche, on y trouvera de nombreuses questions ponctuelles, parfois obstinément accumulées par une même personne, portant souvent sur des points déterminants du dogme catholique et émises sur la base d'un savoir expérimental. On retrouve ainsi souvent l'argument selon lequel les corps humains ne peuvent ressusciter à la fin des temps, puisque chacun peut constater leur putréfaction dans les semaines qui suivent le décès ; la naissance de Jésus sans rapport sexuel préalable fait également l'objet de commentaires qui, formulés dans une perspective essentiellement comique et grivoise, n'en questionnent pas moins le dogme relatif à l'Incarnation. À ce sujet, les femmes interrogées par l'Inquisition ont d'ailleurs souvent une opinion spécifique liée à leur expérience de l'accouchement : est-il vraiment possible que Dieu ait été maculé de sang et de placenta en sortant des entrailles de Marie ${ }^{28}$ ? De telles interrogations n'incitent pas seulement à reconsidérer le rôle des femmes dans le développement de l'incroyance (dont elles sont souvent exclues par les historiens pour des raisons diverses) : elles obligent à prendre en compte l'importance du savoir expérimental, maladroitement formulé mais tenace, dans l'opposition au dogme. Ainsi, à en croire S. Flanagan et D. Weltecke, ce ne 
sont pas les raisonnements des lettrés, mais les observations quotidiennes des petites gens qui auraient alimenté le scepticisme ou l'athéisme; dès lors, pour expliquer le développement de ces courants à la fin du Moyen Âge, la question ne consiste plus à savoir comment ces idées se sont diffusées depuis les élites vers le reste de la population, mais pourquoi ces élites ont alors subitement cessé d'étouffer cette lancinante opposition populaire ${ }^{29}$.

Dans leur chasse méthodique aux idées reçues, les recherches récentes à propos du scepticisme se sont encore emparées d'un autre lieu commun de l'historiographie : celui selon lequel l'athéisme, en tant qu'opposition la plus radicale envers le catholicisme, aurait été systématiquement et impitoyablement pourchassé par l'Église, et que cette menace expliquerait en partie la rareté des déclarations athées explicites ${ }^{30}$. Or en ce domaine également, quelques surprises attendent le lecteur, notamment sous la plume de D. Weltecke ${ }^{31}$.

15 L'auteur rappelle ainsi que le doute n'est généralement pas présenté par les sources comme une agression envers le catholicisme. Il est vrai que celui qui hésitait dans le domaine religieux avait pour lui les figures bibliques des apôtres Pierre et Thomas; agrémentés à la sauce scolastique, ces précédents prestigieux pouvaient faire passer le doute pour un mal nécessaire, voire pour une vertu permettant de renforcer ses convictions à moyen terme. Dans d'autres cas, l'auteur d'un doute ou même l'athée faisaient l'objet de compassion plutôt que de reproches: la desperatio religieuse, antinomique de la foi, était généralement associée à (et, iconographiquement, représentée comme) une faiblesse physique, éventuellement due à un excès de jeûne ${ }^{32}$. Le comportement des intéressés semblait d'ailleurs confirmer ce schéma, puisqu'en plusieurs occurrences, ceux qui perdaient la foi, désespérés par le bouleversement psychologique et social que cela impliquait, étaient tentés par le suicide, confirmant ainsi, dans les catégories médiévales, le caractère pathologique de leur état ${ }^{33}$. On était ainsi bien loin de l'imagerie positiviste du XIXe siècle, dans laquelle le sceptique, héros de l'humanité en voie vers la connaissance, était présenté comme l'incarnation de la vitalité ; l'avantage de cette conception misérabiliste pour les intéressés résidait dans le fait que, aussi longtemps que l'indécision religieuse ne débouchait pas sur une prise de position active et prosélyte, la réaction dominante relevait de la commisération plutôt que de la dénonciation.

En adéquation avec cette perception condescendante, l'arsenal répressif élaboré contre le sceptique ou l'athée était, toujours selon D. Weltecke, étonnamment léger; plus exactement, l'athéisme en tant que tel constituait certes un péché, mais pas un crime juridiquement sanctionnable. Cette constatation étonnante s'explique par le fait que, pour des raisons pratiques, les textes normatifs tels que la législation canonique ou les manuels d'inquisiteurs avaient été conçus contre les déviances perçues comme étant les plus dangereuses, c'est-à-dire celles qui avaient gagné une part importante de la population. Les grilles d'enquête étaient ainsi destinées à identifier les grandes hérésies, donc les croyances en d'autres systèmes religieux que le catholicisme; si un agnostique suspendait totalement son jugement ou si un athée ne professait aucun des dogmes connus, il échappait aux chefs d'accusation préconçus et avait des chances d'échapper aux mailles du filet ${ }^{34}$. Précisons que sur ce point, l'auteur de ces lignes ne partage pas complètement l'optimisme de l'historienne. Certes, l'hérésie et l'athéisme sont des réalités bien différentes du point de vue moderne, et étaient également distingués dans le droit médiéval; mais peut-on affirmer que cette distinction était toujours appliquée 
comme telle par le juge, et celui-ci n'était-il pas enclin à assimiler hâtivement l'athée à l'hérétique, ou encore à l'apostat ou au blasphémateur, également punis de mort depuis Justinien $^{35}$ ? Il reste que l'anomalie décelée par D. Weltecke est effectivement frappante : peut-être parce que les athées avoués étaient trop peu nombreux pour être perçus comme une menace, ils n'étaient pas catalogués explicitement comme une agression envers le catholicisme et faisaient l'objet d'une réprobation et d'une répression bien moins développées que les hérétiques, alors qu'ils défendaient une position plus extrême que ces derniers, de notre point de vue. Ainsi, les historiens de l'époque moderne ou contemporaine qui développèrent l'image de l'athée médiéval systématiquement persécuté réagissaient inconsciemment envers la censure de leur propre époque, mais passaient à côté de l'attitude effectivement adoptée par les autorités du Moyen Âge.

Tels sont donc les principaux acquis de ces trois monographies consacrées au doute ou à l'athéisme. Mais celles-ci présentent encore un autre point commun, puisqu'elles se présentent toutes trois comme des études très inachevées n'ayant été publiées que pour ouvrir la voie à des travaux ultérieurs, et proposent des suggestions en ce sens. Pour compléter le présent bilan de l'historiographie en ce domaine, on résumera donc ici ces pistes de recherche, avant d'y ajouter quelques autres desiderata de la recherche qui n'ont pas été signalés par les trois auteurs.

En premier lieu, si l'on souhaite reprendre le dossier existant ou l'enrichir par de nouvelles sources, que faut-il chercher ou, plus précisément, quelles sont les expressions médiévales qui désignent le doute ou l'athéisme ? Là encore, c'est à D. Weltecke qu'il convient de rendre hommage, puisque celle-ci a méticuleusement sillonné ce champ lexical, qu'elle a contribué à déminer ${ }^{36}$. Elle a ainsi pu souligner d'abord la diversité de ce vocabulaire : aux lemmes classiques « infidel* » ou « incredul* » ainsi qu'à l'adjectif dubiosus , elle propose ainsi d'ajouter l'impietas ou la desperatio, mais également l'acedia, désignant une absence totale d'assiduité religieuse (notamment à la messe) liée au scepticisme. Elle met en garde, toutefois, contre la très grande palette sémantique de ces termes, qui outrepasse parfois franchement les limites de notre sujet. Ainsi, l'infidelitas, au Moyen Âge comme de nos jours, peut s'appliquer au domaine conjugal autant que religieux; de même, accuser quelqu'un de "nier Dieu " peut consister à le qualifier de despote qui bafoue les règles élémentaires de l'humaine société, sans que cela n'indique quoi que ce soit à propos de ses convictions dogmatiques (on se rapproche alors de l'expression moderne « sans foi ni loi ») ; même la notion de «non credere in deum " peut être ambiguë, dans la mesure où elle peut désigner une crainte face aux décisions divines, mais pas nécessairement une négation de l'existence de Dieu. Enfin, l'historienne rappelle qu'en bien des occurrences, une source relative à l'athéisme ne contiendra aucun de ces termes: c'est là une manière de prendre utilement distance vis-à-vis d'une tendance historiographique littéraliste qui rejette la possibilité d'une réalité sociale dès lors que celle-ci n'a pas encore reçu un terme adéquat dans la société considérée. On a vu, en effet, que les sources médiévales savaient évoquer l'athéisme bien avant que le terme ne fût inventé au XVIe siècle, par des périphrases ou d'autres procédés littéraires. En certaines occurrences, un comportement était suffisamment explicite: celui qui dérobait des hosties pour les dévorer chez lui agrémentées d'oignons et de charcuterie ne donnait-il pas clairement son avis à propos de la transsubstantiation ${ }^{37}$ ? Au terme de cette patiente analyse lexicale, le champ de recherches est à la fois étendu et mieux balisé pour des recherches futures. 
19 À cette première piste de recherche, on associera encore trois lacunes qui semblent patentes dans ce champ de recherches, en revenant d'abord sur la question du vocabulaire. Apparemment, aucune des trois études présentées, pas plus que celles qui les ont précédées, n'a recouru aux bases de sources médiévales numérisées pour identifier des extraits concernant le sujet. On rétorquera peut-être que, précisément en raison de l'imprécision lexicale que l'on vient de signaler, la recherche de mots-clés dans de telles bases de données peut se révéler difficile ou franchement vaine; il reste qu'un certain nombre de notions étaient associées, avec une marge d'imprécision certes considérable, au scepticisme dans les sources médiévales, et qu'un tel sondage serait donc vraisemblablement fructueux. Surtout, les outils d'analyse de ces bases textuelles ont considérablement progressé depuis quelques années: loin de ne permettre que la recherche d'un mot ou d'un lemme isolé, ils permettent désormais une mise en contexte $\mathrm{du}$ terme en question, pour déterminer si celui-ci voisine avec d'autres mots issus du champ lexical concerné par l'enquête. On pourrait ainsi isoler les cas dans lesquels infidelis, terme polysémique comme on l'a vu, était bien employé dans un sens religieux et non pas pour une affaire conjugale ou pour qualifier la trahison d'un vassal vis-à-vis de son seigneur. Ces outils de recherche permettraient ainsi d'élargir le corpus médiéval lié au doute ou à l'athéisme, un résultat particulièrement précieux pour ce champ de recherche à propos duquel les historiens se sont souvent contentés, comme on l'a vu plus haut, de ressasser les mêmes dossiers documentaires, alors que ceux-ci étaient lourdement grevés par les malentendus ou les partis pris de l'époque moderne.

Toujours dans l'espoir d'accroître le corpus disponible, les historiens du scepticisme, comme bien d'autres, auraient vraisemblablement intérêt à examiner non seulement les textes originaux produits par le Moyen Âge, mais également ceux, plus anciens, que cette période a transmis : si le De rerum natura de Lucrèce a été copié et annoté dans plusieurs bibliothèques - et ce fut le cas -, on peut admettre que cet héritage a davantage marqué les esprits médiévaux que les problèmes de conscience, même très explicites et actuels, d'une paysanne isolée de Montaillou au XIIIe siècle. Dans le même ordre d'idées, il pourrait être utile de sonder les commentaires médiévaux des textes antiques évoquant le scepticisme : comment la maïeutique de Socrate fut-elle perçue à «l'âge de la foi »? Et comment les exégètes médiévaux répondaient-ils aux moments de doute attribués aux apôtres, ou à la figure du fou vétérotestamentaire qui affirmait l'inexistence de Dieu ? Par ces divers procédés, on gagnerait un volant de sources nouvelles qui, certes, ne concernait probablement que le public lettré, mais qui préciserait notre connaissance à propos des approches théoriques du doute et de l'athéisme au Moyen Âge.

21 Sur un dernier point, enfin, les analyses des ouvrages présentés ici mériteraient d'être élargies pour gagner en précision: on pense à la question de la périodisation. Non seulement les trois auteurs accordent une place très maigre aux évolutions chronologiques durant les quatre siècles qu'ils étudient, mais surtout, la borne initiale qu'ils ont communément adoptée pour leur terrain d'enquête, le début du XIIe siècle, convainc d'autant moins qu'aucun d'entre eux ne la justifie explicitement. Certes, au courant de ce même XIIe siècle, Étienne de Tournai pestait contre un nouveau « vent de savoir séculier » qui posait des questions, absurdes selon lui, à propos de la Trinité, de l'Incarnation ou de la Résurrection ${ }^{38}$; mais il n'était pas le premier qui, au cours de l'histoire humaine, se plaignait d'une recrudescence récente de l'esprit critique, et il semble téméraire d'en déduire l'existence, dans le domaine du scepticisme, d'une « renaissance du XIIe siècle »- un concept historiographique très critiqué par ailleurs. De 
nombreux indices, au contraire, semblent indiquer que le haut Moyen Âge n'avait pas échappé au doute religieux, même si en l'occurrence, l'enquête complète reste à faire. Non seulement l'athéisme était déjà évoqué (pour être condamné, on s'en doute) par des œuvres carolingiennes telles que la Vision de saint Paul du IXe siècle ${ }^{39}$, mais à la même époque, Jean Scot Erigène produisait des théories qui influencèrent peut-être les panthéistes du XIIIe siècle tels qu'Amaury de Bène ${ }^{40}$. Par ailleurs, le haut Moyen Âge avait eu son lot d'anticléricalisme sous des formes diverses, ou de conversions de chrétiens à d'autres religions. Ces deux derniers phénomènes ne constituent certes pas, on l'a vu, une preuve directe pour l'existence du scepticisme ni a fortiori de l'athéisme, mais dans la mesure où ils sont souvent invoqués, en étant rapportés au bas Moyen Âge, comme des signes pratiques de réticence vis-à-vis du dogme catholique, pourquoi exclure de cette analyse les premiers siècles médiévaux ? On ne peut donc que constater, avec un peu d'amusement, que les trois auteurs qui ont contribué à effacer, dans le domaine du doute religieux, l'image moderne d'un Moyen Âge obscurantiste, ont implicitement reporté ce cliché vers le haut Moyen Âge au lieu de changer radicalement de paradigme. Le tableau reste donc à compléter.

En dépit des rares réserves que l'on vient de formuler, on reconnaitra volontiers, en guise de conclusion, que les trois ouvrages présentés ici ont fait progresser, avec une belle unanimité, nos connaissances à propos du doute religieux médiéval et ébranlé bien des préjugés à ce sujet, et que ce feu d'artifice éditorial ouvre une nouvelle phase dans l'historiographie consacrée à ce thème. Rappelons-en une dernière fois les résultats principaux. Il est désormais acquis qu'au Moyen Âge, le scepticisme a existé, les expressions d'athéisme semblent extrêmement vraisemblables et l'agnosticisme est bien attesté. Il est vrai que la perception de ces formes d'incroyance fut très différente, durant cette période, de ce qu'elle allait être à l'époque moderne ou a fortiori à l'ère contemporaine: ne pas croire en Dieu, avant la fin du XVe siècle, constituait une souffrance, et non un crime ou la base d'une théorie ${ }^{41}$. Il n'empêche: ces résultats plaident une nouvelle fois contre une conception trop fixiste de l'idéologie médiévale. Les mentalités étaient manifestement flexibles, y compris dans le domaine religieux; et il en va de même pour l'appareil normatif, qui tolérait une idée quelle qu'elle fût aussi longtemps que celle-ci ne déclenchait pas une innovation d'une ampleur propre à menacer le corps social. Tels sont les résultats méritants et, espérons-le, provisoires, de ces trois beaux livres.

\section{NOTES}

1. BullMHFA, 44, 2008, p. 227.

2. Peter Dinzelbacher, Unglaube im "Zeitalter des Glaubens». Atheismus und Skeptizismus im Mittelalter, Badenweiler : Wissenschaftlicher Verlag Bachmann, 2009 ; Sabina Flanagan, Doubt in an Age of Faith. Uncertainty in the Long Twelfth Century, Turnhout: Brepols (Disputatio, 17), 2008; Dorothea Weltecke, « Der Narr spricht: Es ist kein Gott. » Atheismus, Unglauben und Glaubenszweifel vom 12. Jahrhundert bis zur 
Neuzeit, Frankfurt am Main/ New York: Campus (Historische Studien, 50), 2010. Le premier et le troisième de ces ouvrages ont fait l'objet d'une recension sur le site internet H-Soz-u-Kult : http://hsozkult.geschichte.hu-berlin.de/rezensionen/.

3. Lucien Febvre, Le Problème de l'incroyance au XVIe siècle : la religion de Rabelais, Paris : A. Michel (L'Évolution de l'humanité), 1942.

4. Georges Minois, Histoire de l'athéisme. Les incroyants dans le monde occidental des origines à nos jours, Paris : Fayard, 1998.

5. P. Dinzelbacher, Unglaube ..., op. cit., p. 151-154.

6. D. Weltecke, Der Narr ..., op. cit., p. 23-99.

7. D. Weltecke, Der Narr ..., op. cit., p. 106-123.

8. D. Weltecke, Der Narr ..., op. cit., p. 123-152.

9. D. Weltecke, Der Narr ..., op. cit., p. 152-163.

10. D. Weltecke, Der Narr ..., op. cit., p. 163-180.

11. D. Weltecke, Der Narr ..., op. cit., p. 180-212.

12. D. Weltecke, Der Narr ..., op. cit., p. 230-254.

13. Pierre le Vénérable, De miraculis libri duo, livre II, ch. 25, éd. D. Bouthillier, Turnhout : Brepols (Corpus Christianorum. Continuatio Mediaevalis, 83), 1988, p. 142-46 ; cité par S. Flanagan, Doubt ..., op. cit., p. 166 et suiv.

14. Otloh de Saint-Emmeram, Liber de temptatione cuiusdam monachi, éd. et trad. S. Gäbe, Bern etc. : P. Lang, 1999 (Lateinische Sprache und Literatur des Mittelalters, 29), p. 248-261 ; cité par D. Weltecke, Der Narr ..., op. cit., p. 386 et suiv.

15. L'exemple le plus connu est celui d'Anselme de Canterbury (v. 1033-1109) et de son Proslogion, éd. F.S. Schmitt, S. Anselmi Cantuariensis Archiepiscopi opera omnia, 2e éd., Stuttgart : Fromann, 1984, vol. 1, p. 89-122 ; cité par P. Dinzelbacher, Unglaube ..., op. cit., p. 20-21. Mais des débats équivalents sont menés à l'époque scolastique.

16. D. Weltecke, Der Narr ..., op. cit., p. 394 et suiv.

17. Césaire de Heisterbach, Dialogus miraculorum, distinctio IV, c. XXXIX, éd. J. Strange, Köln/ Bonn/ Bruxelles : Heberle, 1851, p. 206-208.

18. P. Dinzelbacher, Unglaube ..., op. cit., p. 10 et suiv. ; D. Weltecke, Der Narr ..., op. cit., p. 11 et suiv., préconise des distinctions similaires.

19. P. Dinzelbacher, Unglaube ..., op. cit., p. 135 et suiv.

20. Giraud de Barri, Gemma ecclesiastica, 2, 24, éd. J.S. Brewer, J.F. Dimock, G.F. Warner, London : Longman, 1862 (Rerum Britannicarum Medii Aevi Scriptores, 21), p. 285 ; cité par P. Dinzelbacher, Unglaube ..., op. cit., p. 46.

21. Deborah Goodwin, Take Hold of the Robe of the Jew : Herbert of Bosham's Christian Hebraism, Leiden : Brill, 2006, p. 230 ; cité par S. Flanagan, Doubt ..., op. cit., p. 82.

22. S. Flanagan, Doubt ..., op. cit., p. 157-183.

23. Jean de Salisbury, Polycraticus, livre VII, c. 2, éd. Ch.J. Webb, Oxford : Clarendon Press, 1909, vol. II, p. 99 ; cité par S. Flanagan, Doubt ..., op. cit., p. 16.

24. Bernard de Clairvaux, De consideratione, livre V, éd. J. Leclercq, H.-M. Rochais, Sancti Bernardi Opera, vol. III, Roma : Editiones Cistercenses, 1972, p. 471 ; cité par S. Flanagan, Doubt ..., op. cit., p. 106. 
25. Guillaume de Chartres, Appendix vitae Iae s. Ludovici regis, 38, Acta Sanctorum Aug., 5, Paris/ Roma, 1868, p. 567, col. D.

26. P. Dinzelbacher, Unglaube ..., op. cit., p. 147-151.

27. Blanche B. Boyer, Richard McKeon (éd.), Sic et Non : a Critical Edition, Chicago etc. : University of Chicago, 1977 ; cité et analysé par S. Flanagan, Doubt ..., op. cit., p. 139-143.

28. Il s'agit là du cas bien connu d'Aude Fauré à Montaillou, analysé par D. Weltecke, Der Narr ..., op. cit., p. 236 et suiv.

29. D. Weltecke, Der Narr ..., op. cit., p. 431-432. L'auteur rejoint là les conclusions de Susan Reynolds, "Social mentalities and the case of medieval scepticism " in: Transactions of the Royal Historical Society, 6/1, 1991, p. 21-41, ici p. 28.

30. Telle est encore l'opinion défendue par P. Dinzelbacher, Unglaube ..., op. cit., p. 2.

31. D. Weltecke, Der Narr ..., op. cit., p. 309-369.

32. Ce point est souligné également par S. Flanagan, Doubt ..., op. cit., p. 96 et suiv.

33. Un exemple parmi d'autres est fourni par P. Dinzelbacher, Unglaube ..., op. cit., p. 50.

34. D. Weltecke, Der Narr ..., op. cit., p. 335 et suiv.

35. Corpus Iuris civilis, III, Novellae, 77, ch. 1, éd. R. Schoell, W. Kroll, Berlin : Weidmann, 1954, p. 1 ; cité par D. Weltecke, Der Narr ..., op. cit., p. 314.

36. D. Weltecke, Der Narr ..., op. cit., p. 257-309.

37. D. Weltecke, Der Narr ..., op. cit., p. 157.

38. Exemple cité par S. Flanagan, Doubt ..., op. cit., p. 153.

39. Theodore Silverstein (éd.), Visio Sancti Pauli. The History of the Apocalypse in Latin together with nine Texts, London : Christophers (Studies and Documents, 4), 1935, ici p. 143-145 ; cité par P. Dinzelbacher, Unglaube ..., op. cit., p. 121-122.

40. Ce rapprochement, parfois contesté par l'historiographie, est suggéré par P. Dinzelbacher, Unglaube ..., op. cit., p. 25.

41. On reprend là la conclusion de D. Weltecke, Der Narr ..., op. cit., p. 449-467, ici p. 466. 